\title{
A CONCEPTUAL FRAMEWORK FOR AGGREGATE MANAGEMENT OF VIRTUAL ENTERPRISES
}

\author{
Jorge P. Sousa, Américo L. Azevedo, António L. Soares \\ Faculdade de Engenharia da Universidade do Porto and \\ INESC Porto / Manufacturing Systems Engineering Unit, Rua José Falcão 110, \\ 4050-315 Porto, PORTUGAL. \\ Email: \{aazevedo, jsousa, asoares\}@inescporto.pt
}

This paper presents a conceptual framework where the development of an information system for aggregate management of virtual / networked enterprises is taking place. Firstly, the general objectives of the system are described. An ontology based development approach is presented, assuming the role of conceptual guidance, both for the requirements analysis and design phases. Finally, a broad specification of aggregate management in this context is described through use cases.

\section{INTRODUCTION}

Enterprises are increasingly adopting new ways of organisation namely through a shift from traditional vertical integration and towards stronger partnerships leading to Virtual Enterprises or Organisations (GartnerGroup, 1999). In fact, some key features of the new forms of organisation are: intensification of teamwork, integration along the chain, extended just-in-time concept, process integration and organisational knowledge (Apel, 1999).

Traditionally, in a supply-chain, each chain member is a separate entity that may be highly integrated internally but with rigid and linear business processes, when viewed from outside. End-user orders are passed up the chain through each partner and products then flow back down to the end customer in an opposite sequence.

There is a clear conceptual difference between the virtual enterprise model and the vertically integrated model. The virtual enterprise represents, in a certain way, one hybrid or intermediate form of organisation between the concepts of market and hierarchy. In fact, it combines features of a fully market-oriented organisation, such as legal independence of the entities and specialisation of functions, with features of hierarchical forms such as close collaboration with the integration of information and trust between the participating entities.

In this context, we are looking at organisations that are formed on networks of enterprises structured at different levels of a complex chain. Figure 1 illustrates the network environment considered for a core organisation (Org 0.1) with some main 
suppliers (Org 1.1, Org 1.2 and Org 1.3), with different levels of interaction. Furthermore, on the other hand each supplier can be the core company of other complex network.

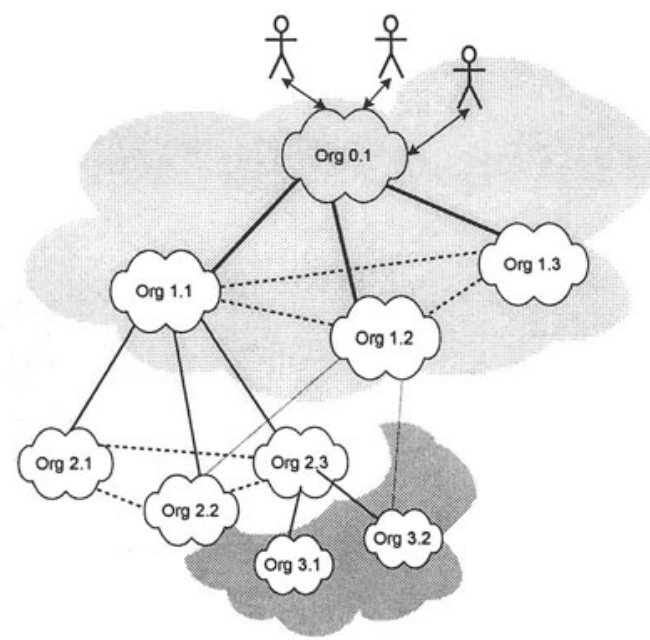

Figure 1 - Network or supply-chain structure

For an effective co-operation and collaboration, these structures need IT infrastructures that support advanced information systems enabling a timely information exchange and full accessability. Furthermore, besides enhancing some the existing electronic commerce transactions (such as EDI), new services should be developed in order to support flexible and non-linear business processes.

\subsection{The Co-OPERATE project}

Co-OPERATE is a project supported by the European Commission, that aims at developing solutions to enhance the entire supply network, providing an advanced information and communication infrastructure to support general co-operation, as well as particular methodologies for co-operative planning and for network set-up and support. The project started in January 2000, and involves two academic groups, a consultancy firm and several industrial companies. The later are part of rather complex and dynamic networks (automotive supplies and electronics).

Business processes have been identified and a conceptual framework and methodologies for aggregate planning are being tested and tailored in the pilot companies. Hopefully they will be widely applicable and easy to adapt to other industries. A Decision Support System for Virtual Enterprises is being developed, but its design runs into large difficulties given the profusion of concepts and terms, and the confrontation of different perspectives. This led to the use of an ontology, as a way to define a conceptual and terminological core, thus implementing a mediation scheme between the users and the the development actors, and allowing knowledge sharing and reuse.

The ontology acts as the conceptual framework guiding the information system development process, from the early requirements analysis phase to the design phase. 
Particular issues to be approached in this process are the transition from a semiformal ontology to the formal and more specialised ontologies to be used by the agents in an agent-based architecture.

The project aims at developing solutions to enhance the entire supply network, from the final product manufacturer to the original material suppliers. It will provide an advanced information and communication infrastructure to support co-operation in dynamic enterprise networks, as well as a methodology for co-operative planning, and a set of mechanisms to enable real-time monitoring of the supply-chain. Moreover, tools to support a fast and effective reconfiguration of manufacturing networks will be made available.

In order to achieve these goals a number of "business solutions" (or general functionalities) have been defined, that will focus on the network aspects of the business processes and will try to accommodate and support the current internal tools and processes of companies. These business solutions are the following (Collings and Loeh, 2000):

Long term business planning for the network - This business solution will improve the long term planning process in the whole supply network. The goal is to generate long-term plans for the network by synchronising forecasts and plans, and by promoting early communication of changes and feedback about feasibility.

Standard operational order and planning processes - This business solution will support the short and medium term operational order and planning processes. This includes order generation and transmission, synchronised planning, monitoring and status information. This business solution should provide a strong basis for real-time order promising and exceptions handling.

Feasibility studies for new order or change requests across the network - This business solution will support the request for new orders or large order changes across the network and will support and co-ordinate the feasibility checks at the individual companies within the shortest possible time. This includes checking of capacity and materials from suppliers.

Exception handling process - This business solution will include methodologies to detect, as early as possible, problems in the network such as peak orders, capacity shortages or part availability problems.

Multi sourcing co-ordination - This business solution will optimise the allocation of work to the different suppliers, based on a defined set of rules and fast negotiation methodologies with the suppliers. It includes a negotiation process on capacities with feedback from the suppliers.

Process visibility - This business solution will develop methodologies to make the progress of orders in the supply network.

Performance management information - This business solution will provide management information about the performance of the network processes. It includes the identification of key performance indicators and provides the methodologies to collect and present them.

\section{A CONCEPTUAL FRAMEWORK FOR CO-OPERATE}

The project consortium comprises partners with different academic and industrial 
cultures. Concerning the industrial partners, the different companies have organisations and languages that can be substantially different. Even if the general objectives and goals of the system under development were set in a precise manner, this was not the case of its scope. The very nature of the project, involving the idea of virtual enterprise, led to difficulties in defining the entities covered by the concept and the roles to be played by those entities. From the point of view of the project management there are obvious difficulties. These co-ordination aspects are in practice an additional problem in terms of an efficient and smooth course of the project.

Our experience showed what others had already reported, in a different context, that terminological confusion breeds conceptual confusion and vice-versa (Bradshaw et al., 1992). This is mostly true in phases of conceptual brainstorming and confrontation of perspectives which characterise the early phases of the system development process. One way to improve this process is to agree a priori with a conceptual and terminological core, setting the ground for the subsequent discussion. Such a core would assume a mediation role between the development actors. This led us to the use of ontologies in implementing the referred mediation scheme.

\subsection{Purpose of an ontology in the systems development life cycle}

Ontologies have been applied so far in a range of works with several purposes. Knowledge sharing and reuse are the base line directly or indirectly referred in each of them. Sharing arises when a common conceptualisation of a given domain is essential to the undertaking and co-ordination of activities within that domain. Sharing implies some sort of communication between different people, people and implemented computational systems, different implemented computational systems (Uschold and Gruninger, 1996b). Reuse can be viewed as the step forward towards a generalised sharing, through formalisation mechanisms. Our purpose in building an ontology for this project is to improve the communication between partners concerning the requirements identification, specification and design phases of the planning and control system. In this way we expect to achieve a faster agreement on the system's conceptual model and a more consistent use of terms and concepts throughout the software development. Eventually, when a more mature state of this ontology is reached, we intend to code it and make it available for reuse, contributing for the clarification of the Virtual Enterprise concept. As a side effect, we also expect to contribute to the research of a less explored role of an ontology: the one of communication medium between people.

In the construction of the Virtual Enterprise ontology (VEo) we followed whenever possible the methodology for developing ontologies outlined by Uschold and King (1995). This methodology includes the following steps: identify purpose, build the ontology (capture, code, integrate existing ontologies), evaluation and documentation. According to our goals, we concentrated in the purpose identification and building steps, particularly the capture phase. The later is probably a crucial step in the process, and consists in identifying the key concepts and relationships in the domain, producing precise text definitions, identifying terms, and reaching an agreement on these issues. Another important aspect in the capture phase is the inclusion/integration of other ontologies. In our case, the Enterprise Ontology 
(Uschold et al., 1996) was particularly helpful and was thoroughly used in the VE Ontology construction. The Plan Ontology (Tate, 1995), although in a draft state, was also used.

\subsection{The VE Ontology as a mediator in the system development life cycle}

As referred above, one less explored role of an ontology is the one of communication medium between different people working together for a given purpose. This is the case of a (software) system development process where teams composed by end users and system developers have to collaborate throughout the development life cycle. In this research work our goal is to extend the communication role towards the mediation of the "world views" of the development actors. In doing so, we intend to improve the engineering of an intrinsically complex software system, particularly in the requirements identification, system specification and system design phases, overcoming some of the difficulties described before.

During the development life cycle world views of end-users and developers constraint the purpose, scope and goals of the development object, as well as the discourse to describe and reason about the domain/system. Though the purpose, scope and goals of the system must be minimally agreed early in the project, conceptual differences and language misunderstandings and redundancies are important obstacles in a smooth evolving of the process.

The degree of influence that each users and developers world views can have in the development life cycle phases is qualitatively represented in the figure by the two areas separated by the thick grey linevariable throughout the process. End-users world-view has more influence in the requirements identification and specification phases whilst developers world view is more influential in the system design phase. System development phases and milestones are shown overlapping each other to symbolise both the fuzziness of the borders between phases and a desirable as the result of a system development approach based on evolutionary prototyping. The role of the ontology as mediator is materialised in the concepts and terminology used in the system development milestones - statement of requirements, specification models and system architecture, which are expressed according to the terminology and structure defined by that ontology.

\subsection{The VE ontology in the operation of the system}

A subset of the VE ontology is "used" in the operation of the Co-OPERATE information system. In fact, as the system architecture follows a software agents approach, the content language used by those agents must be specified in detail. Basically, to interact and to inter-operate effectively, agents need a common understanding of the shared knowledge (content language) and a common communication language (interaction expression language). In this architecture, KQML (Knowledge Query Manipulation Language) was adopted as an interaction expression language, because it provides a great variety of communicative acts well suited to ensure agent sociability, by providing support to implement negotiation, contracting, planning and co-operation. 


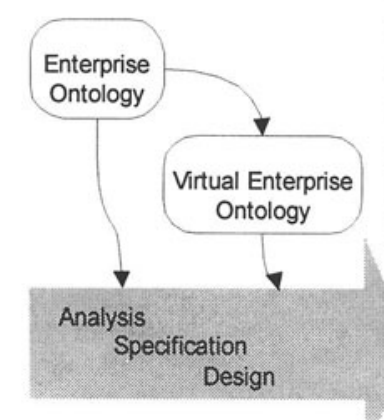

System design

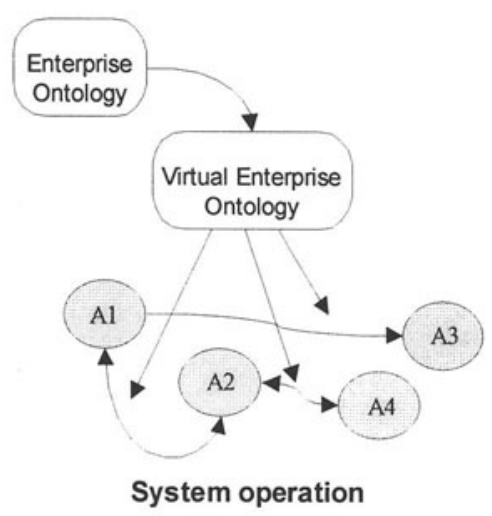

Figure 2 - The role of the VE ontology in the Co-OPERATE life-cycle

The role of a conceptual framework based on the VE ontology is summarised in Figure 2. The left hand side, represents how the VE ontology (including the Enterprise ontology) is used to support the clarification and consistency of concepts throughout the analysis, the specification and the design of the system. The right hand side shows that the agents (in the agent based architecture) exchange messages whose content is subsumed by the VE ontology.

\section{SPECIFICATION OF AGGREGATE MANAGEMENT IN VIRTUAL ENTERPRISES THROUGH USE CASES}

During the analysis phase, a requirements model was defined in order to delimit the system and to define its functionality. The Unified Modelling Language (UML) has been adopted to specify, design and document the system under development (Booch et al., 1999). The UML includes elements that support the creation of several diagrams types. Each diagram provides a different and complementary view of a model of a software system allowing to model all details, regardless of their scope and complexity.

In fact, in a first phase we describe the system as a number of use cases that are performed by a set of actors. The actors constitute the environment of the system, and the use cases specify the functionality from a user's perspective. A use cases model depicts a narrative process that describes the interactions between external actors and the system (an actor is a user of the system in a particular role), and make the system requirements and the terminology used in the domain area more understandable. Use case modelling helps with three of the main phases of development, namely: the capture of requirements, the definition of iterations of development and the validation of system.

For the general functionalities described above "standard operational order and 
planning processes" and "feasibility studies for new order or change requests across the network", the scenario considered assumes that a core company, with their own customers - downstream in the chain, is customer of a number of suppliers. This company holds the order data and ensures the availability of them to their suppliers. The suppliers can request actualisation of orders either by a pull or push process. In Figure 3, we present the use case diagram for an aggregate order process support. We identify four kinds of actors: the customer of the core company, the expeditor at the core company, the supplier and the enterprise information systems (typically an Enterprise Resource Planning system) of the core company.

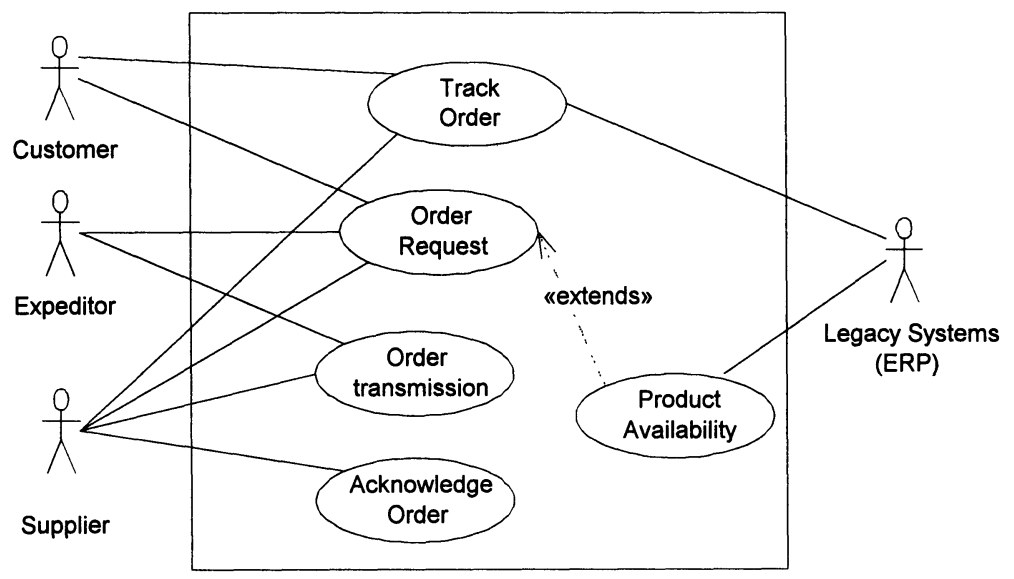

Figure 3 - Use Case diagram for the order process support

For the use case diagram considered, the tasks defined are:

Order transmission - allows to send automatically information to all suppliers using the best communication channel; all orders from the core company to each supplier are presented together with their actual status; the supplier has the choice to view them sorted according to a customisable filter; all new and changed orders (which not yet have been acknowledged by the supplier) are highlighted.

Acknowledgement of orders - the planner of the supplier can acknowledge each new or changed order and automatically the system registers the acknowledgement data such as date/time, user identification, etc; all changes are stored in a history table.

Order request - this task deals with the feasibility of acceptance of new orders, with the production of a quotation, with a real-time check of capacity and materials availability; this task supports and co-ordinates the feasibility checks at the individual companies within the shortest possible time including checking for own capacities and requesting needed materials from suppliers, which need to do the same process themselves; results are consolidated to come to a complete solution; this process achieves a co-operative production plan for the network.

Track Order - basically this unity of functionality allows a end-customer or a supplier, to track, along the production chain, the positioning and the status of 
completion of each related order.

Based on the use cases considered, scenarios are being defined as an instances of the use cases, in order to capture the interactions between the system and all actors involved. These interactions are described as sequences of messages and later, in the implementation phase, will be expressed through the content language and interaction expression language considered.

\section{CONCLUSIONS AND FURTHER WORK}

The interaction among participants in a network of companies, their co-ordination and their access to knowledge are becoming increasingly important. Moreover, setting up the network and managing it in an optimised way, balancing customer needs with increased performance along the whole chain, may be a key factor for the competitiveness of a company. Particularly important is the support to co-operation and collaboration, as well as the definition of a methodology for planning and customer order negotiation business processes.

The work being done under the scope of the European project Co-OPERATE approaches these questions by providing an advanced information and communication infrastructure to support co-operation in networked and distributed organisations. A distributed and decentralised information system, based on an architecture of agents, and extensively using the internet is being designed and implemented, as a means to provide new and more powerful decision support tools for this type of environments. The general process and framework for the development of this system have been briefly described in this paper. Preliminary results are very promising, leading to a first iteration of the requirements specification and system design (Azevedo et al., 2000)

\section{REFERENCES}

1. Appel, W. 1999. Towards the theory of Virtual Organisations: A description of their formation and figure. Newsletter Vol. 2, No. 2, virtual-organisation.net. http://www.virtual-organization.net

2. Azevedo, A., Sousa, J., Soares, A., 2000, Requirements for an agent based information system supporting variably coupled networked enterprises, in E-Business and Virtual Enterprises: Managing Cooperation Towards Smart Organizations, L. Camarinha-Matos, H. Afsarmanesh and R. Rabelo (Eds.).

3. Booch, G., Rumbaugh, J., Jacobson, I., 1999. The Unified Modelling Language User Guide. AddisonWesley.

4. Bradshaw, J.M., Boose, J.H., Shema, D.B., 1992, Steps Toward Sharable Ontologies for Design Rationale. AAAI-92 Design Rationale Capture and Use Workshop, San Jose, CA, July.

5. GartnerGroup 1999. Frictionless E-Business. White paper from Gartner Consulting, USA.

6. Collings, N. and Loeh, H. 2000. Co-OPERATE Project, Solution Scope. Project IST-1999-12259.

7. Tate, A., 1995, Towards a Plan Ontology, Technical Report AIAI - The University of Edinburgh.

8. Uschold, M., King, M., 1995, Towards a Methodology for Building Ontologies. Technical Re-port AIAI-TR-183, The University of Edinburgh.

9. Uschold, M., King, M., Moralee, S., Zorgios, Y. The Enterprise Ontology. Technical Report AIAI-TR195, The University of Edinburgh, 1996. http://www.aiai.ed.ac.uk / entprise/enterprise/

10. Uschold, M., Gruninger M., 1996b, Ontologies: Principles, Methods and Applications. Knowledge Engineering Review, 11 (2). 